\title{
CRITERIA FOR INNER PRODUCT SPACES
}

\author{
RAYMOND W. FREESE
}

1. Introduction. Let $\Sigma$ denote the class of generalized euclidean spaces $E$. The problem of characterizing metrically the class $\Sigma$ with respect to the class $B$ of all Banach spaces has been solved in many different ways, as in [3], [4], [6], and [8]. This paper attacks the problem along the lines exhibited by Blumenthal [2] in the larger environment of the class $\mathfrak{T}$ of complete, metrically convex and externally convex metric spaces. While the Jordan-von Neumann condition has long been used as a standard, showing it is implied by newly postulated conditions, this paper uses instead as criteria the feeble and weak euclidean four point properties of Blumenthal [1] and [2] and shows that they are implied by the four point condition we shall postulate.

2. Classes of quadruples and corresponding four point properties. The following five classes of metric quadruples of distinct points play a role in what follows.

A metric quadruple $p_{1}, p_{2}, p_{3}, p_{4}$ belongs to class

$\mathcal{C}_{1}$ if and only if it contains a linear triple,

$\mathcal{C}_{2}$ if and only if $p_{3}$ is between $p_{2}, p_{4}$ and $p_{2} p_{3}=p_{3} p_{4}$,

$\mathfrak{C}_{3}$ if and only if $p_{2}, p_{3}, p_{4}$ are linear and $p_{1} p_{2}=p_{1} p_{4}$,

$\mathfrak{C}_{4}$ if and only if $p_{3}$ is between $p_{2}, p_{4}$ and $p_{2} p_{3}=p_{3} p_{4}, p_{1} p_{2}=p_{1} p_{4}$,

$\mathfrak{C}_{5}$ if and only if $p_{3}$ is between $p_{2}, p_{4}$ while $p_{2} p_{3}=2 p_{3} p_{4}$ and $p_{1} p_{2}=p_{1} p_{3}$.

We shall use the terminology introduced by Blumenthal and others as follows.

Definition. A metric space has the weak, feeble, isosceles weak, isosceles feeble, external isosceles feeble euclidean four point property provided every quadruple of its points of class $\mathfrak{C}_{1}, \mathfrak{C}_{2}, \mathfrak{C}_{3}, \mathfrak{C}_{4}, \mathfrak{C}_{5}$, respectively, is congruently embeddable in the (two dimensional) euclidean space. A metric space is said to have the queasy euclidean four point property provided that for each two of its points there exists a third point $r$ between them such that for all points $s$ of the space the quadruple $p, q, r, s$ is congruently embeddable in the euclidean space (and hence in the plane).

3. The four point condition in $\mathfrak{T}$. In this paper, we shall show that in the class $\mathfrak{T}$ the external isosceles feeble euclidean four point

Received by the editors April 19, 1967. 
property characterizes those spaces that are generalized euclidean. From [1] it is known that the weak euclidean four point property effects such a characterization and hence, since $\mathfrak{C}_{5}$ is contained in $\mathfrak{C}_{1}$, it suffices to show that in the class $\mathbb{T}$ those spaces possessing the externally isosceles feeble euclidean four point property also possess the weak euclidean four point property. In the discussion that follows, $M$ will denote an arbitrary member of $\mathfrak{T}^{*}$, the subclass of $\mathfrak{T}$ possessing the external isosceles feeble euclidean four point property.

Lemma 1. Each two distinct points of $M$ are the endpoints of exactly one metric segment.

Proof. Suppose the contrary; that is, $p$ is different from $q$ while there exist $S_{1}(p, q), S_{2}(p, q)$ and $r$ denotes a point in $S_{1}-S_{2}$. In traversing $S_{1}$ from $r$ to $p$ a first point $u$ of $S_{2}$ is encountered while a first point $v$ of $S_{2}$ is similarly met in traversing $S_{1}$ from $r$ to $q$. Let $m_{1}, m_{2}$, be the midpoints of the subsegments of $S_{1}, S_{2}$ that have only the endpoints $u, v$ in common.

Consider a segment $S\left(m_{1}, m_{2}\right)$. Either there exists a point $x$ of $S\left(m_{1}, m_{2}\right)$ such that at least one of the distances $u x, v x$ is greater than $\frac{1}{2} u v$ or else $u x=v x=\frac{1}{2} u v$ for all $x$ of $S$. In the latter case, denoting by $s$ the point of $S$ such that $m_{1} s=2 s m_{2}$, it follows that $u, m_{1}, s, m_{2}$ are congruently embeddable in the euclidean plane and hence $u s$ is less than $u m_{2}$ contrary to fact.

If there exists an $x$ such that $u x$ is greater than $\frac{1}{2} u v$ or $v x$ is greater than $\frac{1}{2} u v$, then the maximal value is assumed at some point $t$ of $S$ for which, say, $u t$ is greater than $\frac{1}{2} u v$. Since $u t>u m_{1}=u m_{2}$, then as above there exist first points $w_{1}, w_{2}$ of $S\left(m_{1}, m_{2}\right)$ such that $u w_{1}=u w_{2}$ $=u m_{1}$ encountered in traversing $S$ in each direction from $t$; it follows by continuity that there exist points $y, z$ of $S$ such that $u y=u z>u m_{1}$ and $y z=2 z w_{1}$. Hence $w_{1}, y, z, u$ are isometrically embeddable in the euclidean plane and therefore $u m_{1}$ is greater than $u y$, contrary to fact.

Having established the uniqueness of segments, we now establish the uniqueness of (metric) lines in members of $\mathfrak{M}^{*}$.

LEMMA 2. In the space $M$, metric segments admit unique prolongations.

Proof. Since $M$ is externally convex, the segment $S(p, q)$ may be prolonged while if it admits of two prolongations, say beyond $q$, then by continuity points $r, s$ of different prolongations exist such that $r \neq s, s q^{*}=s r, q^{*} r=2 q^{*} x$ for some $x, q^{*}$ of $S(p, q)$ for which $s$ is not metrically between $q^{*}$ and $r$ but $q^{*}$ is metrically between $x$ and $r$. Then the quadruple $x, q^{*}, r, s$ is congruent with four points $x^{\prime}, q^{\prime}$, 
$r^{\prime}, s^{\prime}$ of the euclidean plane which with $s$ in a prolongation of $S^{\prime}\left(x, q^{*}\right)$ implies the linearity of $x^{\prime}, q^{\prime}, s^{\prime}$, contrary to fact.

Combining the results of Lemmas 1 and 2 we have

LEMma 3. Each two distinct points of $M$ are contained in exactly one metric line.

Lemma 4. Given in $M$ a metric line $L$ and a point $p$ not in $L$, there exists exactly one foot of $p$ in $L$.

Proof. Since it is known that there exists at least one, let $p$ be a point of $M$, not a point of $L$ and let $f_{p}, f_{p}^{\prime}$ denote two distinct feet of $p$ in $L$. If all points of $L$ between $f_{p}$ and $f_{p}^{\prime}$ are feet of $p$ then the quadruple $p, f_{p}^{\prime}, q$ is congruently embeddable in the euclidean plane where $q$ is the point of $L$ between $f_{p}$ and $f_{p}^{\prime}$ such that $f_{p} q=2 f_{p}^{\prime} q$. The embeddability of the quadruple in the euclidean plane implies $f_{p} p$ is greater than $p f_{p}^{\prime}$ contrary to both $f_{p}, f_{p}^{\prime}$ being feet of $p$ in $L$.

If not all points of $L$ between $f_{p}$ and $f_{p}^{\prime}$ are feet, then there exists a point $r$ of $S\left(f_{p}, f_{p}^{\prime}\right)$ such that $p r>p f_{p}$ and $p r \geqq p x$ for all $x$ of $S\left(f_{p}, f_{p}^{\prime}\right)$. Letting $x$ range from $f_{p}$ to $r$ and $y=y(x)$ be the point of $S^{\prime}\left(x, f_{p}^{\prime}\right)$ such that $x y=2 y f_{p}^{\prime}$ it follows as in Lemma 1 (upon consideration of the function $p x-p y)$ that there exist points $x^{*}, y^{*}$ such that $p x^{*}=p y^{*}$ and $x^{*} y^{*}=2 y^{*} f_{p}^{\prime}$. Hence by the external isosceles feeble euclidean four point property, $p f_{p}^{\prime}$ is greater than $p y^{*}$ contrary to the definition of $f_{p}^{\prime}$.

Additional information concerning the "strictly monotone" nature of the distance function is given in the next lemma.

Lemma 5. If $L$ is a metric line of $M, p$ a point of $M$ not in $L, f_{p}$ the foot of $p$ on $L$, and $q, r$ are points of $L$ such that $q$ is between $r$ and $f_{p}$, then $p q$ is less than $p r$.

Proof. Suppose the contrary. Either for some $x$ of $S(q, r), p x-p r$ $\neq 0$, or $p x-p r=0$ for all $x$ of $S(q, r)$. If for some $x, p x-p r \neq 0$, there exist points $s, r^{*}, r^{\prime}$ of $S^{\prime}\left(f_{p}, r\right)$ such that $p r^{*}=p r^{\prime}=k<p s$ and $r^{*}$, $r^{\prime}$ are the first points of $S_{1}(s, r), S_{2}\left(s, f_{p}\right)$ respectively, encountered (beginning at $s$ ) such that their distance from $p$ is $k$, where $s$ is a point of $S^{*}\left(r^{\prime}, r^{*}\right)$ such that $p s \geqq p x$, for all $x$ of $S^{*}\left(r^{\prime}, r^{*}\right)$. As previously, there exist points $x^{*}, y^{*}$ of $S^{*}\left(r^{\prime}, r^{*}\right)$ such that $x^{*} y^{*}=2 y^{*} r^{*}$ while $p x^{*}=p y^{*}$. Hence the quadruple $p, x^{*}, y^{*}, r^{*}$ is congruently embeddable in the euclidean plane and therefore $p r^{*}$ is greater than $p y^{*}$ contrary to $r^{*}, r^{\prime}$ having distance from $p$ no greater than that of any point in $S^{*}\left(r^{*}, r^{\prime}\right)$.

Now suppose $p x=p r$ for all $x$ of $S(q, r)$. Then if $z^{*}$ denotes the point of $S(q, r)$ such that $q z^{*}=2 z^{*} r$, it follows as above that the 
quadruple $p, q, r, z^{*}$ is congruently embeddable in the euclidean plane and hence $p r$ is greater than $p z^{*}$ contrary to fact.

The development thus far will now allow us to determine the location of the foot of the "vertex" of the isosceles triple in a quadruple of class $\mathfrak{C}_{3}, \mathfrak{C}_{4}$, or $\mathfrak{C}_{5}$ on the line determined by the remaining points of the quadruple.

LeMma 6. In $M$, if $p$ is a point not lying on a line $L$ and if $q, r$ are points of $L$ with $p q=p r$, then $m$, the midpoint of $q$ and $r$ is the foot of $p$ in $L$.

Proof. By Lemma 5 and Lemma 4, the foot of $p$ lies between $q$ and $r$. Suppose $f_{p} \neq m$ and assume the labeling such that $f_{p}$ lies between $q$ and $m$. Given the numbers $m q, m f_{p}$, it follows there exists a positive integer $k$ and a positive real number $\epsilon$ such that $m q-m f_{p}+\epsilon<2^{k} \epsilon$ $<m q+m f_{p}-\epsilon$. Now points $s, t$ can be found such that $f_{p}$ is between $s$ and $t$ while $p s=p t$ and $s t=2 \epsilon$. Then the midpoint of $s, t$ has distance less than $\epsilon$ from $f_{p}$. Using $p s=p t$ it follows that $2 k$ applications of the external isosceles feeble euclidean four point property will show the existence of points $u, v$ of the line such that $p u=p v$ while $q$ is between $f_{p}$ and $u, v$ between $f_{p}$ and $r$, thus contradicting, by Lemma 5 , the fact that $p q=p r$.

While it is not evident that $\mathfrak{C}_{4}$ is contained in $\mathfrak{C}_{5}$, the following lemma shows that the assumption of embeddability in the euclidean plane of all quadruples of $\mathfrak{C}_{5}$ implies, in $\mathfrak{T}$, such embeddability of the members of $\mathfrak{e}_{4}$.

Lemma 7. The space $M$ possesses the isosceles feeble euclidean four point property.

Proof. Consider a point $p, f_{p}$ its foot on a metric line $L(q, r)$ where $p q=p r$, and $p^{\prime}, q^{\prime}, r^{\prime}$ is a triple of points of the euclidean plane congruent to the triple $p, q, r$, while $f_{p^{\prime}}$ denotes the foot of $p^{\prime}$ on $L\left(q^{\prime}, r^{\prime}\right)$. Then, noting that $f_{p}$ is the midpoint of $q, r$, let $r_{n}, q_{n}$ be respective midpoints of $S\left(f_{p}, r_{n-1}\right), S\left(f_{p}, q_{n-1}\right)$ where $q_{0}=q$ and $r_{0}=r$. Then since Lemma 5 and Lemma 6 together with continuous monotoneity of the distance function $p x, x$ in $L(q, r)$, imply $p r_{n}=p q_{n}$ for all $n$, it follows by repeated applications of the external isosceles feeble euclidean four point property and distance properties of the euclidean plane that $p^{\prime} r_{n}^{\prime}=p r_{n}=p q_{n}$ for all $n$ where $r_{n}^{\prime}$ is the point corresponding to $r_{n}$ under the obvious correspondence of $L(q, r)$ with $L\left(q^{\prime}, r^{\prime}\right)$. Since $\left\{r_{n}\right\}$ converges to $f_{p}$ and $\left\{r_{n}^{\prime}\right\}$ converges to $f_{p^{\prime}}$, it follows that $\left\{p r_{n}\right\}$ converges to $p f_{p}$ and $\left\{p^{\prime} r_{n}^{\prime}\right\}$ converges to $p^{\prime} f_{p^{\prime}}$, which with $p r_{n}=p^{\prime} r_{n}^{\prime}$ for all $n$ implies $p f_{p}=p^{\prime} f_{p^{\prime}}$. 
The next two lemmas establish similar results relating $\mathfrak{C}_{5}$ with $\mathfrak{C}_{2}, \mathfrak{C}_{1}$.

Lemma 8. The space $M$ has the isosceles weak euclidean four point property.

Proof. Let $p, q, r$ be points of $M$ such that $p q=p r$ and let $s$ be any point on a line $L(q, r)$. As a result of Lemma 7 , we may assume $s \neq f_{p}$, the foot of $p$ on $L$. Letting $p^{\prime}, q^{\prime}, r^{\prime}$ denote a triple of points of the euclidean plane congruent to $p, q, r$, it follows by Lemma 7 that $p f_{p}=p^{\prime} f_{p^{\prime}}$ where $f_{p^{\prime}}$ is the foot of $p^{\prime}$ on $L\left(q^{\prime}, r^{\prime}\right)$. Letting $s^{\prime}$ denote the point corresponding to $s$ under the obvious congruence of $L(q, r)$ with $L\left(q^{\prime}, r^{\prime}\right)$, it follows, by applying Lemma 7 to the quadruple $p, f_{p}, s, t$ (where $t$ is the point of $L(q, r)$ distinct from $s$ such that $p s=p t$ ), that the quadruple is congruent with a quadruple $p^{*}, f_{p^{*}}, s^{*}, t^{*}$ of the euclidean plane. Then from $f_{p^{*}} s^{*}=f_{p^{\prime}} s^{\prime}$ and $p^{*} f_{p^{*}}=p^{\prime} f_{p^{\prime}}$ as well as the Pythagorean Theorem in the euclidean plane it follows that $p s=p^{*} s^{*}$ $=p^{\prime} s^{\prime}$. Hence the quadruple $p, q, r, s$ is congruent with the quadruple $p^{\prime}, q^{\prime}, r^{\prime}, s^{\prime}$ of the plane and the theorem is proven.

Lemma 9. The space $M$ possesses the weak euclidean four point property.

Proof. Let $p, q, s, u$ be any four points of $M$ such that $q, s, u$ lie on a metric line $L$ with $p$ not an element of $L$. Assume the labeling such that $q \neq f_{p}$. Then there exists a point $r$ of $L$ such that $p q=p r$. Hence by Lemma $8, p, q, r, s$ are congruently embeddable in the euclidean plane and $p, q, r, u$ are likewise embeddable. Since the images of $p, q$, $r$ form a metric basis for the euclidean plane, the linearity of $q, r, s, u$ implies that $p, q, r, s, u$ are congruently embeddable in the plane also.

TheOREM. The space $M$ is generalized euclidean.

Proof. By Lemma 9 the weak euclidean four point property is equivalent to the external isosceles feeble euclidean four point property in $\mathfrak{T}$. Therefore, by a result of [1], the conclusion follows.

It is worth noting that in Banach spaces the embedding of the class $\mathfrak{C}_{5}$ of quadruples in the euclidean plane is equivalent to the norm relation $\left\|b^{\prime}\right\|^{2}=\left\|b-b^{\prime}\right\|^{2}+3\|b\|^{2}$ holding for every pair of points $b$, $b^{\prime}$ of the space for which $\left\|b-b^{\prime}\right\|=\left\|3 b-b^{\prime}\right\|$.

It would be interesting to know whether the converse of Lemma 7 is valid in $\mathfrak{T}$. That is, if a space of the class $\mathfrak{T}$ possesses the isosceles feeble euclidean four point property, does it possess the external isosceles feeble euclidean four point property, thus being generalized 
euclidean? A similar question can be raised concerning the "isosceles" analogue of the queasy euclidean four point property.

\section{REFERENCES}

1. L. M. Blumenthal, Theory and applications of distance geometry, Clarendon, Oxford, 1953.

2. - An extension of a theorem of Jordan and von Neuman, Pacific J. Math. 5 (1955), 161-167.

3. M. M. Day, Some characterizations of inner product spaces, Trans. Amer. Math. Soc. 62 (1947), 320-337.

4. - On criteria of Kasahara and Blumenthal for inner product spaces, Proc. Amer. Math. Soc. 10 (1959), 92-100.

5. M. Fréchet, Sur la definition axiomatique d'une classe d'espaces vectoriels distancies applicables vectorellement sur l'espace de Hilbert, Ann. of Math. 36 (1935), 705-718.

6. P. Jordan and J. von Neumann. On inner products in linear metric spaces, Ann. of Math. 36 (1935), 719-723.

7. H. Rubin and M. Stone, Postulates for generalizations of Hilbert space, Proc. Amer. Math. Soc. 4 (1953), 611-616.

8. I. J. Schoenberg, $A$ remark on $M$. M. Day's characterization of inner product spaces and a conjecture of L. M. Blumenthal, Proc. Amer. Math. Soc. 3 (1952), 961-964.

9. W. A. Wilson, $A$ relation between metric and euclidean spaces, Amer. J. Math. 54 (1932), 505-517.

\section{SAINT LOUIS UNIVERSITY}

